\title{
Carbon Fibre/Silicon Composite Sensors for Concrete Structural Health Monitoring in Compression
}

\author{
Lili Yang ${ }^{a}$, Yongquan Zhang ${ }^{b}$, Yong $\mathrm{Ge}^{\mathrm{c},{ }^{,}}$, Qinghua Zhu ${ }^{\mathrm{d}}$, and Ce Zhang ${ }^{\mathrm{e}}$
}

School of Transportation Science and Engineering, Harbin Institute of Technology, Harbin 150090, P.R. China

aliliyang@hit.edu.cn, ${ }^{b}$ zyqzygzs@126.com, ${ }^{c,}{ }^{*}$ hitbm@163.com, ${ }^{d}$ qinghuazhu2005@126.com roronoazoroshx@126.com

Keywords: Structural Health Monitoring; Concrete; Carbon Fibre/Silicon Composite; Compression

\begin{abstract}
Structural health monitoring of concrete infrastructures has attracted enormous attention due to the brittle nature of concrete. In this research, we report the carbon fibre/silicon rubber composite sensors and their excellent monitoring in concrete's compression. It is shown that the electric resistance change synchronously with the mechanical deformation of concrete during the compression process. With the increase of carbon fibre fraction, the sensitivity decreases and the numerical equations to predict the structural change are also obtained. The experimental results reported here highlight the potential application of $\mathrm{CF} /$ silicon composites as an effective, real-time structural monitoring sensor with low-cost and long life.
\end{abstract}

\section{Introduction}

Structural health monitoring (SHM) and damage detection tools have been urgently needed for civil infrastructures/systems due to the increased awareness of economic and social effects of deterioration resulting from the coupling effect of long-term, fatigue and mutation [1-3]. Among the conventional materials for infrastructures, cement-based concrete is of typical brittle materials and there are large number of randomly distributed micro-cracks and micro voids inside. Under the external force, the micro-crack will continue to expand and finally link to each other $[4,5]$. Compared with other kinds of SHM sensors such as optical fibre or piezoelectric materials [6, 7], conductive polymer composites (CPC) are of increasing importance and show promising properties for a wide range of sensor principles. The major reason is the possibility to tailor the material properties very precisely and they also show outstanding flexibility, low cost, high durability and great compatibility with structural materials [8-10]. Among these materials, composites with carbon fibers as dopants have been intensely studied due to their excellent electric and mechanical properties. Carbon fibre/silicon rubber composites as structural health monitoring sensors were studied in our group and it has been proved that the composites have stable electric resistance-time characteristic, wide working temperature range and large deformation [11]. In this work, their real-time structural monitoring in concrete's compression was further studied. Results show that they have excellent sensing performance.

\section{Experimental}

Preparation of $\mathbf{C F} /$ silicon rubber composites. Short carbon fibres (CF) were chosen with conductivity of $5.6 \times 10^{4} \mathrm{~S} / \mathrm{m}$ and length of $3 \mathrm{~mm}$. They were cleaned with acetone and kept in the oven. As the matrix, silicon rubber was diluted with hexane. The composite sensors were prepared by room temperature melt mixing method [11]. Carbon fibres were added into the diluted solution and mixed manually and then under the ultrasonic device. Four weight fractions of $0.6 \%, 0.75 \%, 0.8 \%$ and $0.9 \%$ were chosen, which corresponds to the volume fraction of $0.84 \%, 1.05 \%, 1.13 \%$ and $1.26 \%$. The residual air in the mixture could be removed through evacuating device. Organotin and 
tetraethoxysilica (TEOS) were added under mixing which were used as the catalytic and crosslinking agent, respectively. After $24 \mathrm{~h}$ room temperature curing in a mold, the composites were obtained with a size of $150 \mathrm{~mm} \times 150 \mathrm{~mm} \times 3 \mathrm{~mm}$.

Characterizations. Samples were coated with platinum and observed on a QUANTA 200F (FEI, America) at an accelerating voltage of $20 \mathrm{kV}$. The sensitivity tests of composites were characterized by the real-time combination of mechanical and electronic performance. Concretes doped with polymer were chosen as the study goal. The four-electrode method was used in the resistance tests which were carried on a digital multimeter (UT805, Uni-Trend Group Limited). The strain and stress results were obtained with the help of CSS- 88000 series electronic universal testing machine.

\section{Results and Discussion}

Morphologies of composites. Fig. 1 shows the typical pictures of composites with low and high fraction of carbon fibres. It indicates that carbon fibres after treatments are uniformly distributed in the silicon rubber. Moreover, with the increase of carbon fibres, the bridging points between fibres increase which form the channels for electron transport in the whole composites.
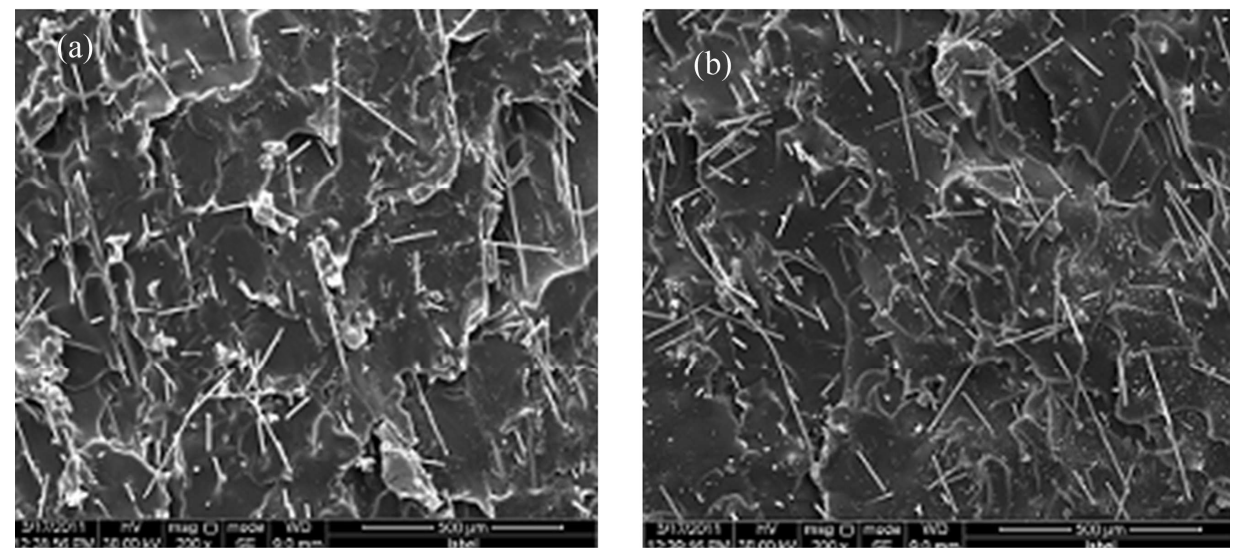

Fig. 1 Typical SEM images of carbon fiber/silicon composites with doped fraction of $0.84 \%$ (a) and $1.26 \%$ (b)

The electrical property of $\mathrm{CF} /$ silicon rubber composites is firstly studied before the sensing performance. Fig. 2 shows the current-potential curves of composites. It is obvious that the composites agree with the Ohm's law when the potential is smaller than $8 \mathrm{~V}$, which is similar with some metallic conductors. However, the conductivity is slightly increased due to the decrease of CF's resistance as the temperature rises during the electric process. As a matter of fact, the composites will expand simultaneously which increase the distance between fibres and decrease the conductivity of composites. According to the results from Fig. 2, it represents that the latter effect is not obvious here.

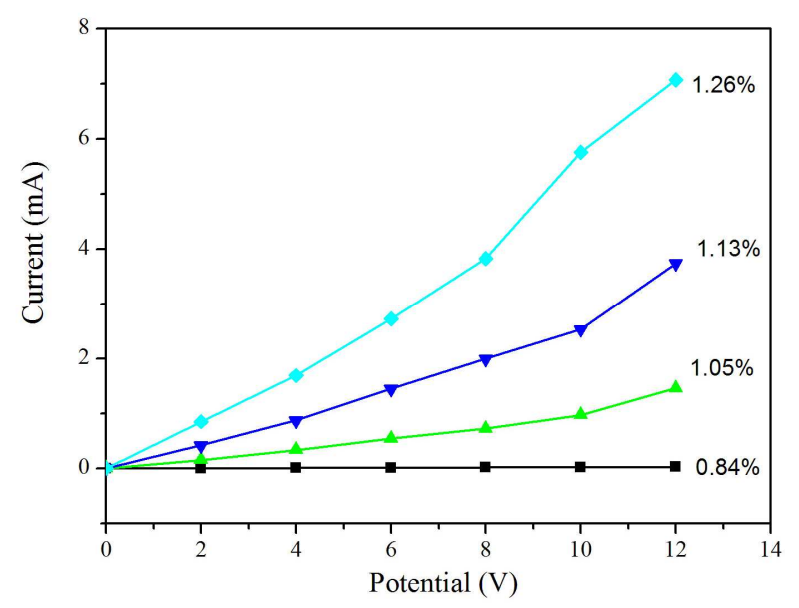

Fig. 2 The current-potential curves of $\mathrm{CF} /$ silicon composites with different volume fraction of $\mathrm{CF}$ 
Structural monitoring properties. The composites were bonded to the concrete using the post-yield epoxy. Electrical contacts are established by drying colloidal silver paste over single-strand wire to form a two-probe measurement setup as shown in Fig. 3. After a $6 \mathrm{~h}$ drying period, the structural monitoring of $\mathrm{CF} /$ silicon rubber sensors during concrete's compression is conducted by combination of compressive load tests and electric characterization.
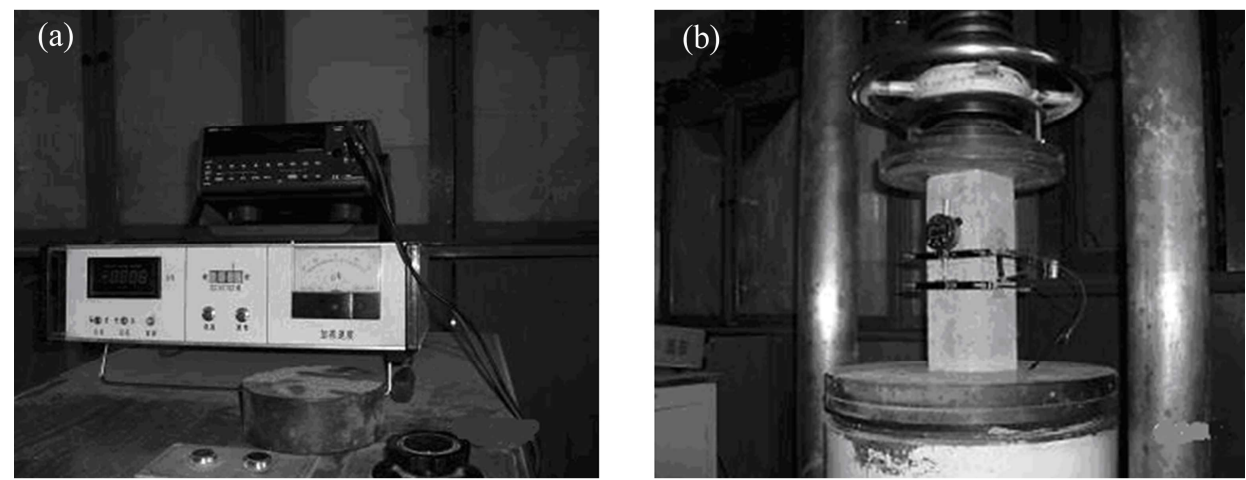

Fig. 3 Equipments (a) and testing (b) images of $\mathrm{CF} /$ silicon sensors monitoring the concrete compression

The compressive strain, stress of concrete and the electric resistance of composites were collected until the failure of concrete. It is observed that the four side faces of concretes expand due to the longitudinal compression and the sensors were tensed. Fig. 4 shows the experimental results of concrete and composites with different factions. It is shown that stress-strain curves of concretes could be divided into two phases, namely the elastic (OA) and plastic $(\mathrm{AB})$ phase. Meanwhile the electric resistance curves are also divided into two periods $\left(\mathrm{OA}_{1}\right.$ and $\left.\mathrm{A}_{1} \mathrm{~B}\right)$ followed along with stress-strain curve. In the first phase, the stress and electric resistance change ratio linearly increase with the strain. But the resistance changes not greatly in this period. During the second phase, the resistance changes frequently and nonlinearly. The micro-cracks expand inside the concrete until the failure of concrete. The results here indicate that the $\mathrm{CF} /$ silicon rubber composite sensors could monitor the structural change during the compression process.
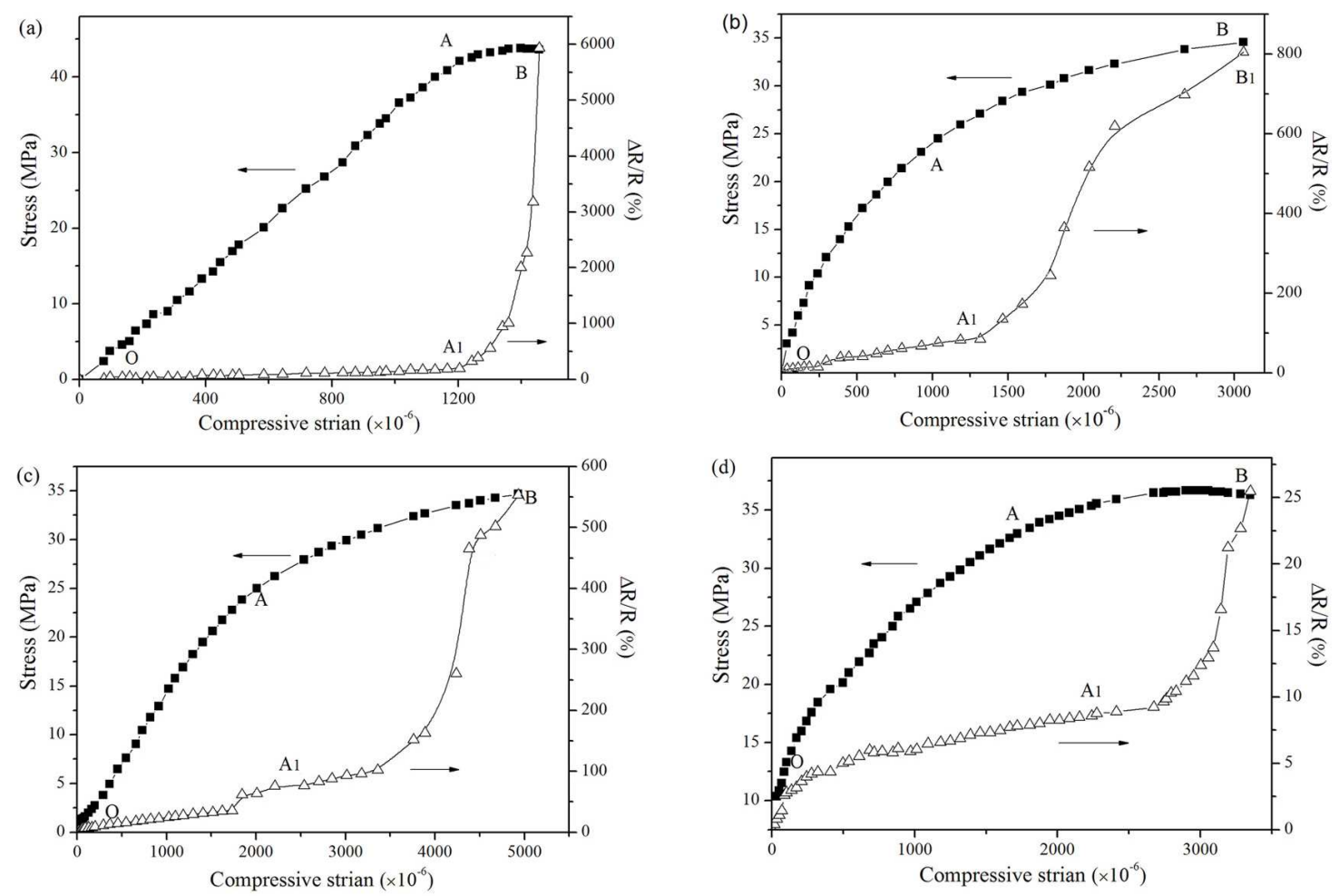

Fig. 4 Sensing curves of composites with different CF fraction $0.84 \%$ (a), $1.05 \%$ (b), $1.13 \%$ (c) and $1.26 \%(\mathrm{~d})$ 
Numerical studies of sensing performation. In order to further describe the sensitivity of $\mathrm{CF} / \mathrm{silicon}$ rubber composite numerically, the resistance change curves at two phases are fitted seperately. It is obvious that a linear relationship could be established for the first phase and an exponential curve is appropriate for the second phase. The first equation is described as:

$$
\triangle R / R=A \varepsilon \quad\left(0<\varepsilon \leq \varepsilon_{0}\right)
$$

where $A$ is the coeffiency or the slope of the fitted lines. Fig. 5 shows the fitted curves for the first phase. It is observed that the slope decreases with the increase of fraction of carbon fibre.

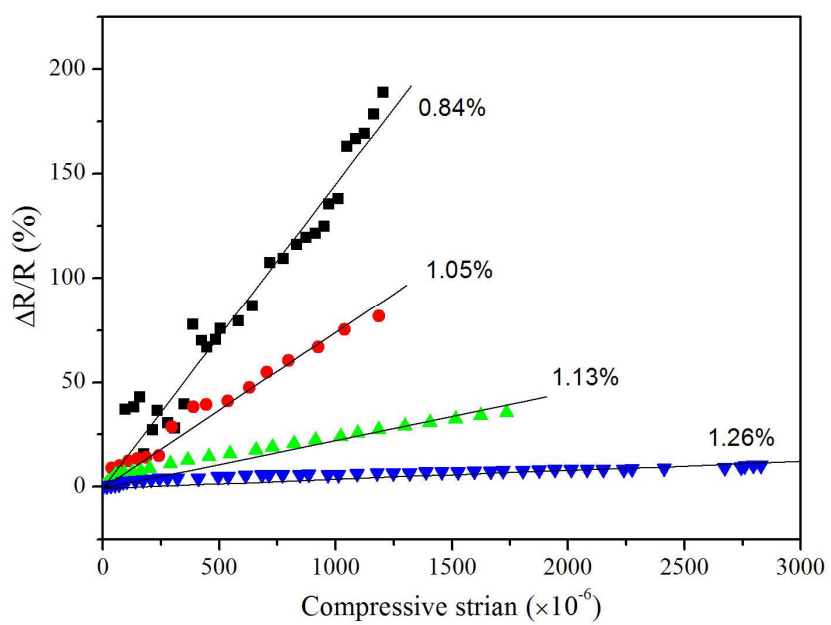

Fig. 5 The experimental and fitted curves about resistance change ratio and compressive strain of composites during the first phase

For the structural health monitoring sensors, one of the most important factors is the strain sensitivity which is expressed as [12]:

$$
S_{R}=\frac{\Delta R / R}{\varepsilon}
$$

Thus $S_{R}=A$ here which means $A$ represents the strain sensitivity. Table 1 shows the calculated results. The sensitivity decreases by two orders of magnitude of composites with the increase of carbon fibre.

Table1 Related fitted parameter during the first phase

\begin{tabular}{ccc}
\hline $\begin{array}{c}\text { Concentration of CF } \\
(\mathrm{vol} \%)\end{array}$ & Parameter $A$ & $\begin{array}{c}\text { Coefficient of correlation } \\
R^{2}\end{array}$ \\
\hline 0.84 & $1.40 \times 10^{5}$ & 0.96 \\
1.05 & $7.06 \times 10^{4}$ & 0.98 \\
1.13 & $2.28 \times 10^{4}$ & 0.98 \\
1.26 & $3.90 \times 10^{3}$ & 0.89 \\
\hline
\end{tabular}

For the second phase, the fitted equation could be described as:

$$
\Delta R / R=C+D \exp (E \varepsilon) \quad\left(\varepsilon_{0}<\varepsilon<\varepsilon_{\max }\right)
$$

where $C, D$ and $E$ are coefficients for the exponential curves. Fig. 6 reveals the fitted results and Table 2 shows the calculated factors for the equations. It is demonstrated that the mechanical formation of concrete during compression could be predicted from these equations easily. 

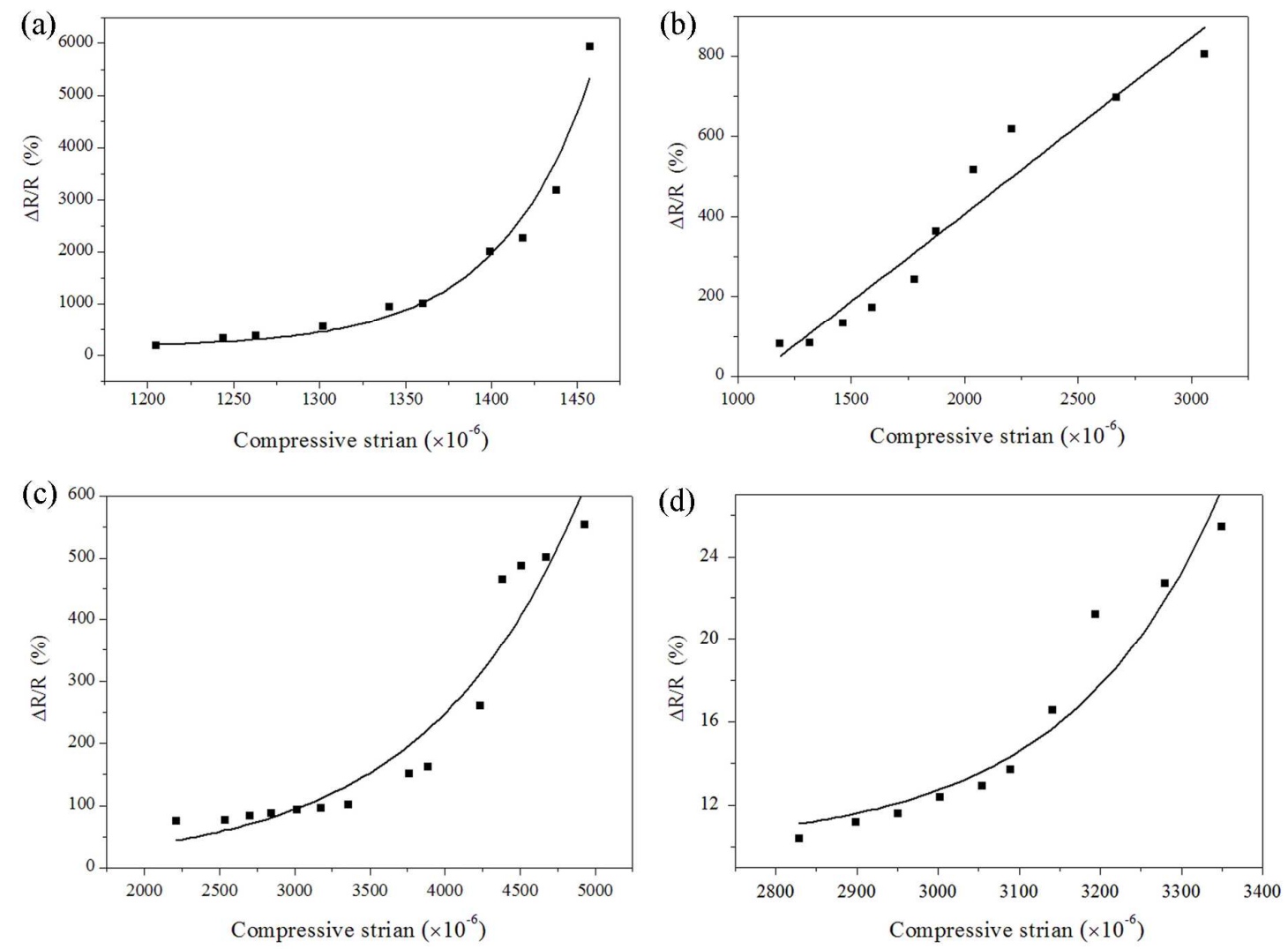

Fig. 6 The experimental and fitted curves of composites with different CF fraction $0.84 \%$ (a), $1.05 \%$ (b), $1.13 \%$ (c) and $1.26 \%$ (d) during the second phase

Comparing with these two phases, the monitoring of the latter one is more important due to the heavier destruction. Thus the broad range at second phase is good for the structural health monitoring. According to the results shown in Fig. 4, the optimal fraction of carbon fibre is about $1.10 \mathrm{vol} \%$. Based on the relationship between the conductivity of composites and their CF fraction, the percolation threshold is $0.80 \mathrm{vol} \%$ [11]. The sensing performance shown here proves that $\mathrm{CF} /$ silicon rubber composites have excellent structural monitoring properties during concrete compression.

Table 2 Related fitted parameters during the second phase

\begin{tabular}{ccccc}
\hline $\begin{array}{c}\text { Concentration of CF } \\
(\mathrm{vol} \%)\end{array}$ & $C$ & $D$ & $E$ & $\begin{array}{c}\text { Coefficient of } \\
\text { correlation } R^{2}\end{array}$ \\
\hline 0.84 & 152.49 & $1.33 \times 10^{-8}$ & $1.83 \times 10^{4}$ & 0.97 \\
1.05 & $1.58 \times 10^{6}$ & $-1.58 \times 10^{6}$ & -0.28 & 0.94 \\
1.13 & 1.25 & 4.884 & $9.81 \times 10^{2}$ & 0.93 \\
1.26 & 10.03 & $3.06 \times 10^{-7}$ & $5.33 \times 10^{3}$ & 0.92 \\
\hline
\end{tabular}

\section{Summary}

In this work, the carbon fibre reinforced silicon composites were prepared and their structural health monitoring of concrete in compression was studied. Results show that the electric conductivity of carbon fibre/silicon rubber composites has the synchronous change with the compressive 
deformation of concrete. The fitted equations in order to predict the structural change are obtained through segmentation fitting. With the increase of carbon fibre fraction, the sensitivity decreases but the monitoring range expands. The optimal fraction is about $1.10 \mathrm{vol} \%$ of carbon fibre.

\section{References}

[1] P.C. Chang, A. Flatau, S.C. Liu, Review paper: health monitoring of civil infrastructure, Struct. Health Monit. 2 (2003) 257-267.

[2] M. Majumder, T.K. Gangopadhyay, A.K. Chakraborty, K. Dasgupta, D.K. Bhattcharya, Fibre Bragg gratings in structural health monitoring-Present status and applications, Sens. Actuators, A 147 (2008) 150-164.

[3] N.D. Alexopoulos, C. Bartholome, P. Poulin, Z. Marioli-Riga, Structural health monitoring of glass fiber reinforced composites using embedded carbon nanotube (CNT) fibers, Comp Sci. Tech. 70 (2010) 260-271.

[4] H. He, P. Stroeven, M. Sroeven, L.J. Sluys, Influence of particle packing on elastic properties of concrete, Mag. Concrete Res. 64(2) (2012) 163-175.

[5] P. Li, H.C. Gu, G.B. Song, R. Zheng, Y.L. Mo, Concrete structural health monitoring using piezoceramic-based wireless sensor networks, Smart Struct. Syst. 6 (2010) 731-748.

[6] H. Inada, Y. Okuhara, H. Kumagai, Health monitoring of concrete structures using self-diagosis materials, Civ. Struct. Health Monit. 4 (2005) 239-248.

[7] G. Song, Y.L. Mo, K. Otero, H. Gu, Health monitoring and rehabilitation of a concrete structure using intelligent materials, Smart Mater. Struct. 15 (2006) 309-314.

[8] M. Wolf, R. Schmittgens, A. Nocke, D. Hecker, M. Liepelt, E. Schulthei, Plasma deposition of conductive polymer composites for strain sensor applications, Prcedia Chem. 1 (2009) 879-882.

[9] C. Cedric, K. Vladan, L. Maryline, Design and development of a flexible strain sensor for texitile structures based on a conductive polymer composite, Sensors 7 (2007) 473-492.

[10] N. Hu, Y. Karube, M. Arai, T. Watanabe, C. Yan, Y. Li, Y. Liu, Investigation on sensitivity of a polymer/carbon nanotube composite strain sensor, Carbon 48 (2010) 680-687.

[11] L.L. Yang, Y. Ge, Q.H. Zhu, C. Zhang, Z.P. Wang, P.H. Liu, Experimental and numerical studies on the sensitivity of carbon fibre/silicone rubber composite sensors, Smart Mater. Struct. 21 (2012) 035011(5pp).

[12] K.J. Loh, J. Kim, J.P. Lynch, N.W.S. Kam, N.A. Kotov, Multifunctional layer-by-layer carbon nanotube-polyelectrolyte thin films for strain and corrosion sensing, Smart Mater. Struct. 16 (2007) 429-438. 